\title{
Scaling Behavior of Fragment Shapes
}

\author{
F. Kun, ${ }^{1}$ F. K. Wittel, ${ }^{2}$ H. J. Herrmann, ${ }^{3}$ B. H. Kröplin, ${ }^{2}$ and K. J. Måløy ${ }^{4}$ \\ ${ }^{1}$ Department of Theoretical Physics, University of Debrecen, P.O. Box 5, H-4010 Debrecen, Hungary \\ ${ }^{2}$ ISD, University of Stuttgart, Pfaffenwaldring 27, D-70569 Stuttgart, Germany \\ ${ }^{3}$ ICP, University of Stuttgart, Pfaffenwaldring 27, D-70569 Stuttgart, Germany \\ ${ }^{4}$ Department of Physics, University of Oslo, P.O. Box 1048-Blindern, 0316 Oslo, Norway
}

(Received 26 June 2005; published 19 January 2006)

\begin{abstract}
We present an experimental and theoretical study of the shape of fragments generated by explosive and impact loading of closed shells. Based on high speed imaging, we have determined the fragmentation mechanism of shells. Experiments have shown that the fragments vary from completely isotropic to highly anisotropic elongated shapes, depending on the microscopic cracking mechanism of the shell. Anisotropic fragments proved to have a self-affine character described by a scaling exponent. The distribution of fragment shapes exhibits a power-law decay. The robustness of the scaling laws is illustrated by a stochastic hierarchical model of fragmentation. Our results provide a possible improvement of the representation of fragment shapes in models of space debris.
\end{abstract}

PACS numbers: 62.20.Mk, 46.50.+a, 64.60.-i

Spacecrafts and satellites, during their mission and service time, are exposed to the danger of impact with pieces of space debris, which is a growing population of rocket bodies, nonfunctioning spacecrafts, rocket fuel ejecta, and pieces of fragmented material accumulated during 40 years of space exploration [1]. In order to minimize the potential hazard, objects of size larger than $10 \mathrm{~cm}$ are continuously tracked in space and their orbits are taken into account for space activities. Fragmentation events, like on-orbit explosions of fuel containers of upper rocket stages and secondary breakups of fragments due to mutual collisions, are the main source of the proliferation of space debris, creating a large number of small fragments which cannot be tracked. For safety reasons it is essential to work out models of fragmentation, i.e., the breaking of objects into smaller pieces, which are able to predict the consequences of on-orbit explosions and impacts [2]. The NASA breakup model EVOLVE05 [2], also implemented by other space agencies, represents the fragments in terms of their characteristic length $L_{c}$, surface-to-mass ratio $A / m$, and velocity $\vec{v}$. Other quantities like the fragment mass $m$ are determined from scaling relations. Model calculations are performed in a phenomenological way; i.e., based on experiments and on-orbit observations of breakup events, probability distributions of the above quantities are prescribed. Monte Carlo simulations are carried out taking into account the specific initial conditions of the event studied [2]. The orbits of fragments are determined by their velocity; however, the lifetime of the orbits is limited by the atmospheric drag which mainly depends on the shape of the fragments. The probability of impact of debris pieces with a spacecraft and the resulting damage can be calculated from their velocity, mass, size, and shape. The precision and predictive power of model calculations strongly rely on the quality of the input distributions and the validity of scaling relations used.
General studies on fragmentation phenomena mostly focused on the understanding of the mass distribution $F(m)$ of fragments. For bulk solids, power-law fragment mass distributions $F(m) \sim m^{-\tau}$ have been obtained under widely varying conditions with universal exponents $\tau$ depending mainly on the spatial dimension $d$ [3-6]. Recently, we have pointed out that the fragmentation of shell-like objects, like fuel containers or rocket bodies relevant for space debris, forms an independent universality class $[7,8]$, which is also supported by other studies [9].

In this Letter we present a study of the shape of fragments generated by explosive and impact loading of closed shells. High speed imaging investigation of the explosion process showed that the shape of fragments is determined by the underlying cracking mechanism of shells which strongly depends on material properties and geometry of the shell: fragments of isotropic shape are obtained for materials where the branching-merging mechanism of cracks governs the breakup, while the formation of long straight cracks results in fragments of a high degree of anisotropy. Fragments of anisotropic shape proved to have self-affine character described by a scaling exponent. The distribution of fragment shapes has a power-law decay with a material dependent exponent. We illustrate the robustness of the scaling laws of the shape of shell fragments by a hierarchical stochastic model. Our results suggest a possible improvement of the description of fragment shapes in phenomenological breakup models of space debris by taking into account the effect of the cracking mechanism of different shell materials on fragment shapes.

In order to understand how the breakup mechanism of shells determines the shape of fragments, we have carried out explosion experiments of closed shells made of brittle materials such as hen eggshells and hollow glass spheres. Apart from the anisotropic shape of the eggs, the geometrical structure of eggshells and glass spheres characterized by the wall thickness ( $w \approx 0.3 \mathrm{~mm}$ ) to radius $(R \approx 2.5 \mathrm{~cm})$ 
ratio $w / R \approx 0.005$ is practically the same. However, their material properties show large differences giving rise to different cracking mechanisms under dynamic loading conditions: the eggshell is composed of a bioceramics characterized by the Young modulus $Y \approx 55 \mathrm{GPa}$, a high damping of elastic waves, and a strongly disordered porous microstructure with a relatively low mechanical strength [10]. Glass is an amorphous material with much higher fracture strength and lower acoustic damping than eggshells [11]. A Photron APX ultima high speed camera with frame rate $15000 / \mathrm{s}$ and spatial resolution $256 \times 256$ pixels was used to follow the time evolution of the explosion process, which also enabled us to study the dynamics of crack formation on the surface, and for the first time in the literature provided direct access to the mechanism of fragmentation. The shells were filled with a stoichiometric hydrogen-oxygen mixture which was electrically ignited approximately in the center of the shell. The analysis of the explosion of 20 eggshells showed that the breakup process starts with the nucleation of a few cracks at the flatter end of the egg (see Fig. 1). The propagation of cracks is driven by the expansion of the shell, i.e., the cracks do not run at a high speed, they advance as the in-plane deformation of the expanding shell gradually increases. Based on the high speed images in Fig. 1, the estimated crack speed falls in the range $v_{c} \approx 150-300 \mathrm{~m} / \mathrm{s}$ which is an order of magnitude below the calculated Rayleigh wave speed $c_{R}=2200 \mathrm{~m} / \mathrm{s}$ of the shell material. Propagating cracks undergo a sequential branching process, which results in a hierarchical treelike crack pattern [Fig. 1(a)] [12]. Fragments are formed along the main cracks by the merging of adjacent sidebranches at almost right angles [Fig. 1(c)] similarly to two-dimensional bulk solids of disordered microstructure [3]. We note that the area of fragments generated at this stage does not show large variations; it is practically determined by the inherent length scale of crack tip splitting. The branching-merging process is initially governed by the in-plane deformation of the shell; however, as the expansion increases the out-ofplane deformation dominates giving rise to further cracks mainly perpendicular to the former ones [13] [Fig. 1(d)]. This cracking proceeds again in a sequential manner, typically breaking the fragments into two pieces until a stable configuration is reached.

Experiments on exploding hollow glass spheres revealed a substantially different breakup mechanism which can be

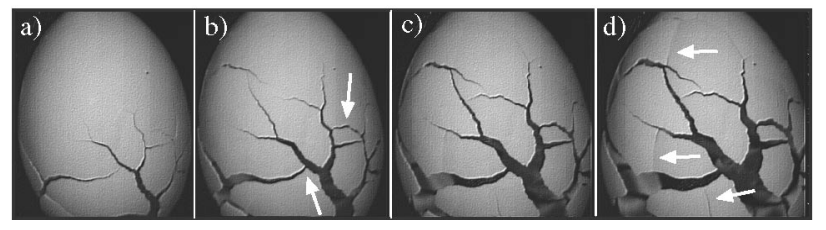

FIG. 1. Consecutive snapshots of an exploding hen eggshell at a frame-rate $15000 \mathrm{1} / \mathrm{sec}$. Merging of sidebranches of the hierarchical crack tree and cracks leading to secondary breakup are indicated by arrows in (b) and (d), respectively. attributed to the different material properties: the breakup of the shell starts at some hot spots with random position on the surface [Fig. 2(a)] mainly determined by fluctuations of the wall thickness. Cracks radiating from hot spots propagate at a high speed $\left(v_{c}>2000 \mathrm{~m} / \mathrm{s}\right)$ close to the Rayleigh wave speed $\left(c_{R} \approx 3300 \mathrm{~m} / \mathrm{s}\right)$ covering large distances without any apparent branching [14] [Figs. 2(a) and 2(b)]. This cracking mechanism results in a large number of long thin fragments having also a relatively large curvature which makes them unstable against bending Fig. 2(c). During the expansion of the sphere these primary fragments undergo a sequential breakup process due to the out-of-plane bending deformation [13] [Fig. 2(c)]. Eggshells and hollow glass spheres were also fragmented by impact with a hard wall, which produced the same type of fragments [8].

In the final state of the breakup process, the fragments were carefully collected and digitized with a scanner for further evaluation. It can be observed in Figs. 1 and 2 and in the inset of Fig. 3 that for the different types of materials considered, the fragments are always compact twodimensional objects with little surface roughness; however, their overall shape can vary from completely isotropic (eggshell) to highly anisotropic (glass) depending on the cracking mechanisms. The mass $m$ and surface $A$ of fragments is defined as the number of pixels $N$ and the contour length of the spots in the digital image, respectively. We characterize the linear extension of fragments by their radius of gyration as $R_{g}^{2}=(1 / N) \sum_{i \neq j=1}^{N}\left(\vec{r}_{i}-\vec{r}_{j}\right)^{2}$, where the sum goes over the $N$ pixels $\vec{r}_{i}$ of the fragments. In order to reveal how the shape of fragments varies with their size, in Fig. 3 the average fragment mass $\langle m\rangle$ is presented as a function of $R_{g}$ for different materials from impact and explosion experiments. It is important to note that in all cases power-law functional forms $\langle m\rangle \sim R_{g}^{\alpha}$ are obtained with a high quality; however, the exponent $\alpha$ depends on the structure of the crack pattern. Since the eggshell pieces have regular isotropic shape, their mass increases with the square of $R_{g}$ and hence $\alpha=2 \pm 0.05$ was fitted. The large glass fragments are characterized by a significantly lower value of the exponent $\alpha=1.5 \pm 0.08$, while for small glass pieces one observes a crossover to isotropic shape with $\alpha=2 \pm 0.08$. The value $\alpha<2$ implies that the frag-

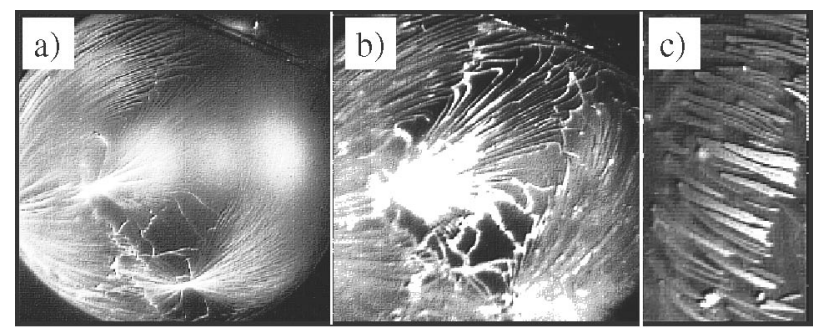

FIG. 2. Snapshots of an exploding hollow glass sphere at a frame rate $150001 /$ sec. Hot spots can be seen in (a) radiating a large number of straight cracks (white lines) (b). Zoom on needlelike fragments is shown in (c). 


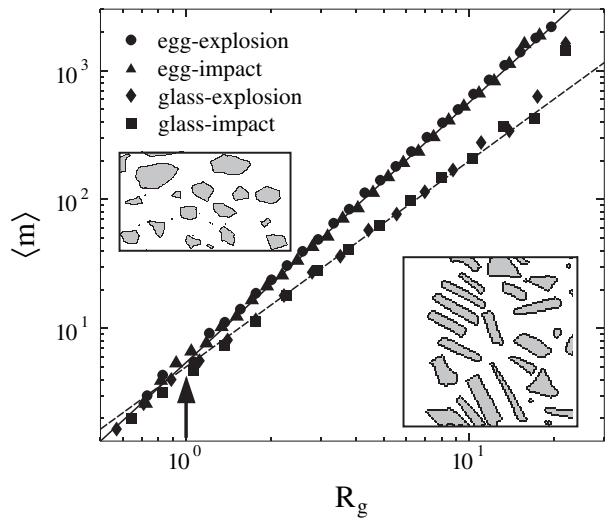

FIG. 3. Average fragment mass $\langle m\rangle$ as a function of the radius of gyration $R_{g}$. The arrow indicates the crossover point of glass fragments. Scanned pieces of egg (left) and glass (right) demonstrate the different fragment morphologies.

ments have self-affine character, i.e., the larger they are, the more elongated they get. Note that similar anisotropy and self-affinity of fragment shapes was not observed in $d$ dimensional bulk fragmentation $(d=2,3)$ [3-6]. To quantify this behavior, let us consider that the shell fragments have a rectangular shape with side lengths $a$ and $b$, hence, the surface, mass, and radius of gyration can be obtained as $A=2(a+b), m=a b$, and $R_{g}=\sqrt{a^{2}+b^{2}} /(2 \sqrt{3})$, respectively. The fragment mass can be expressed in terms of the aspect ratio $r=a / b$ and $R_{g}$ as $m \sim R_{g}^{2} /(r+1 / r)$, where even for moderately elongated fragments the approximation $m \sim R_{g}^{2} / r$ is valid. Consequently, for fragments with exponents $\alpha<2$, the aspect ratio must increase as a power of $R_{g}$ so that $r \sim R_{g}^{\delta}$. Hence, $m \sim R_{g}^{2-\delta}$ follows, and $\alpha=2-\delta$, where $\delta \approx 1 / 2$ was obtained in the experiments. It is interesting to note that the value $\delta=$ $1 / 2$ has been found in a broad class of systems producing self-affine structures, for instance, for the scaling of the width with the length of the arms of noise reduced diffusion limited aggregation clusters, for clusters of directed percolation, or for the Hölder exponent of one-dimensional random walks [15].

Apart from the shape of individual fragments, it is also important to know the probability of occurrence of a specific fragment shape in the final state of a breakup process. The NASA breakup model characterizes the shape of fragments by the surface-to-mass ratio $A / m$, the distribution of which is fitted by a linear combination of Gaussian distributions [2]. The functional form of the corresponding distributions $g(A / m)$ of our shell pieces in the inset of Fig. 4 again shows a strong dependence on the cracking mechanism. For isotropic fragments a reasonable fit could be obtained with Gaussians in agreement with the NASA model [2]; however, for anisotropic fragments $g$ increases monotonically. The small sized isotropic and the large very elongated anisotropic fragments both have large $A / m$ value which prevents clear shape identification. To obtain a better characterization of fragment shapes, we

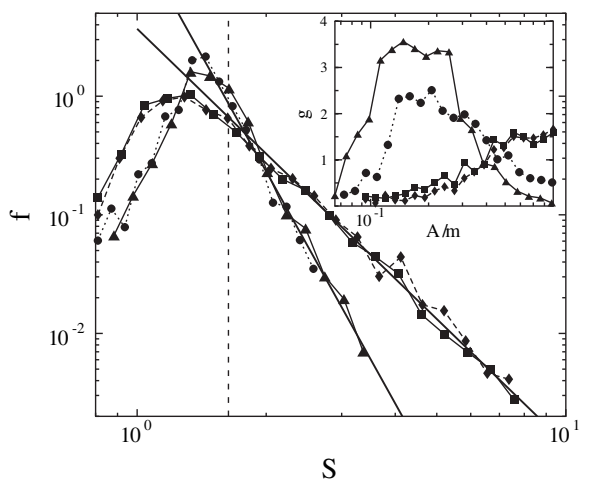

FIG. 4. Distribution $f$ of the shape parameter $S$. Power-law decay is evidenced with different exponents for isotropic and anisotropic fragments. Inset: distribution $g$ of $A / m$ used by the NASA model. The legend is the same as in Fig. 3.

introduce a dimensionless shape parameter $S$ defined as $S=\frac{A}{m} R_{g}$, multiplying the surface-to-mass ratio $A / m$ by the radius of gyration $R_{g}$. Assuming rectangular objects, the shape parameter $S$ takes the form $S=(a+b) \times$ $\sqrt{a^{2}+b^{2}} /(\sqrt{3} a b)$. For fragments of isotropic shape $a \approx$ $b$, it follows that $S \approx 1.63$, which is indicated by the vertical dashed line in Fig. 4. If the fragments are elongated $a \gg b$, the shape parameter $S \approx a / b$ coincides with the aspect ratio $r$ characterizing the degree of anisotropy. Corresponding to the cracking mechanisms, the distributions $f(S)$ of different materials and fragmentation modes (explosion and impact) form two groups in Fig. 4. Fragments of a low degree of anisotropy, irrespective of their size, contribute to the maximum of $f(S)$ in the vicinity of $S \approx 1.63$. Since eggshell fragments are mostly isotropic at all sizes, the distribution $f(S)$ decreases rapidly over a narrow interval of $S$. Although we only have data over less than two decades their precision is sufficiently high to allow proposing a power-law behavior of the distribution $f(S) \sim S^{-\beta}$, where the exponent $\beta=6.8 \pm 0.3$ is obtained for isotropic fragments, while $\beta=3.5 \pm 0.2$ follows when the cracking mechanism favors the formation of anisotropic fragments.

Discrete element models of shell fragmentation usually consider highly disordered brittle materials and provide isotropic fragment shapes, but they have difficulties to capture microscopic mechanisms resulting in long straight cracks $[7,8]$. We propose a simple stochastic binary breakup model in the spirit of Refs. $[4,13,16]$ to better understand the experimental findings. The model focuses on the binary breakup of fragments formed by the primary cracking mechanism of the shell. Representing the fragments by rectangles with a continuous mass distribution at a fixed aspect ratio $r=a / b$, the effect of the primary cracking mechanism on the shape of fragments can be taken into account by setting $r \approx 1$ for fragments of isotropic shape (egg), and $r \gg 1$ for the highly anisotropic needlelike pieces (glass). Based on Figs. 1 and 2, these fragments are then assumed to undergo a sequential binary 


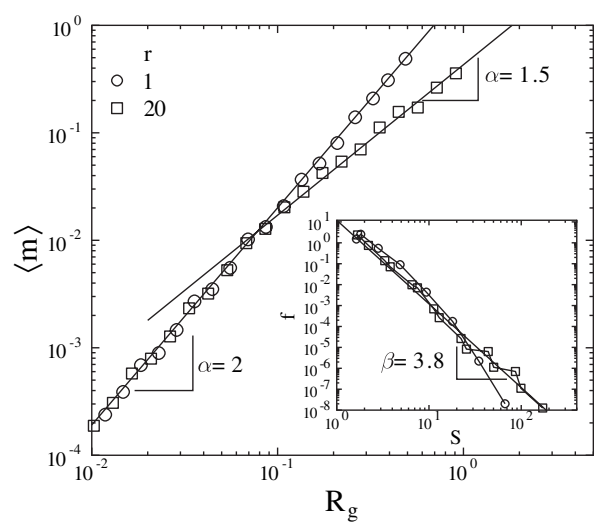

FIG. 5. Log-log plot of $\langle m\rangle$ as a function of $R_{g}$. Inset: distribution $f$ of the shape parameter $S$. Satisfactory agreement is obtained with the experimental results presented in Figs. 3 and 4.

breakup process, where at each step of the hierarchy they break into two pieces of equal mass with a probability $p \leq$ 1 . Note that the fragments have $1-p$ chance to keep their actual size. To capture the effect of out-of-plain deformations, we choose a side of a rectangle to break with a probability proportional to its length $[4,16]$. Computer simulations of the model were performed starting from a continuous distribution of fragment sizes varying the initial aspect ratio $r$ to model different materials, while $p$ was fixed. The hierarchical process was followed up to $n=30$ generations resulting in $\sim 10^{7}$ fragments in the final state, where the mass $m$, the radius of gyration $R_{g}$, and the shape parameter $S$ of fragments were determined. It can be observed in Fig. 5 that similar to the experiments, for all values of $r$ the average fragment mass exhibits a power-law dependence on the radius of gyration $\langle m\rangle \sim R_{g}^{\alpha}$. Starting the process with isotropic shapes $r \approx 1$, the fragments remain isotropic at all levels of the hierarchy implying $\alpha=$ $2 \pm 0.05$ as it was observed for the egg pieces. Modeling glass fragments by a high initial anisotropy $r \gg 1$, the crack mostly occurs along the same side of the rectangle lowering $r$; however, when the two sides become comparable $r$ fluctuates about one. Consequently, large fragments are characterized by an exponent $\alpha$ significantly lower than 2 , while for small pieces a crossover occurs to isotropic shape with $\alpha=2$. In the experiments we estimated the initial aspect ratio of glass fragments from Fig. 2 to fall in the range of $15 \leq r \leq 35$. Simulations with these aspect ratios $r$ proved to provide values of the exponent $\alpha$ in the vicinity of 1.5. In Fig. 5 the results are presented for $r=20$ where $\alpha=1.5 \pm 0.06$ was obtained. When the fragments initially have an isotropic shape, the hierarchical process gives rise to a rapidly decreasing distribution of the shape parameter $f(S)$ over a narrow range as it was observed for egg pieces. Starting the simulation with elongated fragments ( $r=20$ in Fig. 5) the distribution $f$ shows a powerlaw decay $f(S) \sim S^{-\beta}$ with an exponent $\beta=3.8 \pm 0.3$ very close to the experimental value of glass fragments. It is very important to notice that these results for the exponent $\alpha$ and for the distribution of fragment shapes are practically independent on $p$.

Summarizing, based on high speed imaging techniques we have determined the fragmentation mechanism of closed shells: after the primary cracking mechanism of the shell governed by the in-plane deformation, a hierarchical secondary breakup process sets in due to out-of-plane deformations. Contrary to bulk systems, the shape of shell fragments shows large variations from completely isotropic to highly anisotropic fragments depending on the primary cracking mechanism. We pointed out that the anisotropic fragments have a self-affine character with a scaling exponent $\delta=1 / 2$. To give a quantitative characterization of fragment shapes we proposed a shape parameter, the distribution of which was found to exhibit a powerlaw decay. A hierarchical stochastic breakup model provided quantitative agreement with the experimental findings, which demonstrates the robustness of the scaling laws of fragment shapes in shell fragmentation. The results imply that the characterization of fragment shapes in breakup models of space debris production should be improved by a clear distinction of bulk and shell fragmentation and by using scaling laws with exponents depending on the cracking mechanism of the material.

The authors are grateful to $\mathrm{H}$. Klinkrad and C. Wiedeman of ESA for valuable discussions. This work was supported by SFB381. F. K. was supported by OTKA T049209, M041537, and by the Gy. Békési Foundation.

[1] C. A. Belk et al., Meteoroids and Orbital Debris: Effects on Spacecraft, NASA RP-1408 (NASA, Washington, DC, 1997); N. L. Johnson, Adv. Space Res. 5, 11 (1985).

[2] N. L. Johnson et al., Adv. Space Res. 28, 1377 (2001); J. Bendisch et al., Adv. Space Res. 34, 959 (2004).

[3] J. A. Åström et al., Phys. Rev. Lett. 92, 245506 (2004).

[4] D. L. Turcotte, J. Geophys. Res. 91, 1921 (1986).

[5] T. Kadono, Phys. Rev. Lett. 78, 1444 (1997); T. Kadono and M. Arakawa, Phys. Rev. E 65, 035107 (2002).

[6] F. Kun and H. J. Herrmann, Phys. Rev. E 59, 2623 (1999); H. Katsuragi, D. Sugino, and H. Honjo, Phys. Rev. E 68 , 046105 (2003).

[7] F. K. Wittel, F. Kun, H. J. Herrmann, and B.-H. Kröplin, Phys. Rev. Lett. 93, 035504 (2004).

[8] F. K. Wittel, F. Kun, H. J. Herrmann, and B.-H. Kröplin, Phys. Rev. E 71, 016108 (2005).

[9] R. Linna, Ph.D. thesis, University of Jyväskylä, 2004.

[10] N. MacLeod, in Proceedings of the ICF11 Conference (University of Torino, Torino, Italy, 2005), p. 872.

[11] A. Yuse and M. Sano, Nature (London) 362, 329 (1993).

[12] J. Feinberg and M. Marder, Phys. Rep. 313, 1 (1999).

[13] R. Linna et al., Phys. Rev. E 72, 015601 (2005).

[14] J. A. Åström and J. Timonen, Phys. Rev. Lett. 79, 3684 (1997).

[15] K. Kassner and E. Brener, Phys. Rev. E 50, 2161 (1994); H. Hinrichsen, Adv. Phys. 49, 815 (2000).

[16] G. Hernandez and H. J. Herrmann, Physica (Amsterdam) 215A, 420 (1995). 